\title{
Comparisons of Test-Retest Reliability of Strength Measurement of Gluteus Medius Strength between Break and Make Test in Subjects with Pelvic Drop
}

\author{
In-Cheol Jeon \\ Department of Physical Therapy, College of Life \& Health Science, Hoseo University, Asan, Korea
}

\begin{abstract}
Purpose: The purpose of this study was to compare the reliability of unilateral hip abductor strength assessment in side-lying with break and make test in subjects with pelvic drop. Hip abduction muscles are very important in the hip joint structures. Therefore, it is essential to evaluate their strength in a reliable way.

Methods: Twenty-five subjects participated in this study. Unilateral isometric hip abductor muscle strength was measured in side-lying, with use of a specialized tensiometer using smart KEMA system for make test, of a hand held dynamometer for break test. Coefficients of variation, and intra class correlation coefficients were calculated to determine test-retest reliability of hip abductor strength.

Results: In make test, maximal hip abductor strength in the side-lying position was significantly higher compared with break test $(p<0.05)$. Additionally, Test-retest reliability of hip abductor strength measurements in terms of coefficients of variation $(3.7 \%$ for make test, $16.1 \%$ for break test) was better in the side-lying position with make test. All intraclass correlation coefficients with break test were lower than make test ( 0.90 for make test, 0.73 for break test).

Conclusion: The side-lying body position with make test offers more reliable assessment of unilateral hip abductor strength than the same position with break test. Make test in side-lying can be recommended for reliable measurement of hip abductor strength in subjects with pelvic drop.
\end{abstract}

Keywords: Break test, Hip abductor, Make test, Strength measurement

\section{INTRODUCTION}

The gluteus medius (Gmed) muscle is very important in maintaining the stabilization of hip joint. ${ }^{1}$ The Gmed acts as a hip abductor and as a dynamic stabilizer of hip joint, especially during a singlelimb stance and walking ${ }^{2}$ and side-lying position. ${ }^{3}$ Insufficient hip abductor muscle may result in a Trendelenburg gait. ${ }^{4}$

Pelvic drop (PD) is defined as dropping occurrence in pelvis due to Gmed weakness of weight bearing side in one leg standing position. ${ }^{1,4}$ PD can be caused by insufficient Gmed muscle strength as a positive Trendelenburg sign. ${ }^{4}$ The optimal Gmed strength is required to maintain the height of the top of iliac crest in one leg standing position. The Gmed strength of weight bearing side in one leg standing

Received May 7, 2019 Revised Jun 7, 2019

Accepted Jun 11, 2019

Corresponding author In-Cheol Jeon

E-mail jeon6984@hoseo.edu position is tested for Trendelenburg sign. The weakness of the tested side of Gmed contributed to the pelvic drop in one leg standing position. The tested side of Gmed in side-lying position was investigated the lumbopelvic stabilization with core muscles. ${ }^{3}$ In addition, the findings of the previous study reported that the pelvic height was increased in the subject with weakness of Gmed in side-lying. ${ }^{3}$ Therefore, Excessive changes in the Gmed strength cause functional limitations and movement impairments during standing and side-lying. ${ }^{1}$

A hand held dynamometer (HHD) is a common tool used to clinically measure muscle strength. ${ }^{5,6}$ The advantages of HHD include a quick tool of providing objective values in clinic and experimental settings. However, Schwartz ${ }^{7}$ reported that the HHD is less sensitive for graded more than 4 . In addition, between examiners,

Copylight (C)2019 The Korean Society of Physical Therapy

This is an Open Access article distribute under the terms of the Creative Commons Attribution Non-commercial License (Http:// creativecommons.org/license/by-nc/4.o.) which permits unrestricted non-commercial use, distribution, and reproduction in any medium, provided the original work is properly cited. 
correlation coefficients for lower-extremity testing were much lower than those for upper-extremity testing. The grade of inter-examiner for hip abductor was 0.74 , whereas for shoulder flexor was $0.94 .^{8} \mathrm{~A}$ poor correlation coefficient contributed to a poor values of association between repeated tests. Some previous studies concluded that HHD was not reliable to measure the lower-extremity. On the other hand, Hip abductor performed in side-lying position can be measured by HHD with using a long lever arm. ${ }^{5,9}$

Hip abduction in side-lying position is the functional position for testing the strength and performance of the Gmed. As an effective evaluation of the Gmed is an important component in successful rehabilitation of individuals with PD, the precise strength measurement is required in clinical and research settings. The HHD can be used for break test by providing manual resistance during active muscle contraction. The tensiometer can be used for make test by performing the motion in non-elastic band. The more optimal body posture for the assessment of hip abductor strength was investigated as a side-lying position compared to supine and standing. ${ }^{2}$ However, no prior study has compared the test-retest reliability of strength measurement of the Gmed between break and make tests in subjects with PD. The purpose of the present study was to determine the testretest reliability of break test and make test for the strength measurement of the Gmed in a side-lying position. We hypothesized that the reliability would be better in the side-lying position with make test because of more stable counter balanced resistance than break test. The results of this study would guide the prefer measurement regarding the clinical techniques for testing Gmed performance.

\section{METHODS}

\section{Subjects}

$G^{\star}$ Power software was used (ver. 3.1.2, University of Kiel, Germany) in a pilot study of seven participants. The calculation of the sample size was conducted with a power of 0.80 , an alpha level of 0.05 , and an effect size of 1.41. This result indicated that the required sample size for the study was at least fifteen participants. Twenty-five subjects with PD aged 20-30 years were enrolled in this study (Table 1). Inclusion criteria included: (1) normal range of motion in hip joints, (2) no musculoskeletal and neurological dysfunctions, (3) no history of hip joint surgery, (4) a positive sign of Trendelenburg test. Exclusion criteria included: (1) specific hip joint pain such as osteoarthri-
Table 1. General characteristics of the subjects

\begin{tabular}{lc}
\hline & Mean \pm SD \\
\hline Age (year) & $24.2 \pm 3.2$ \\
Height $(\mathrm{cm})$ & $171.4 \pm 3.1$ \\
Weight $(\mathrm{kg})$ & $68.3 \pm 5.2$ \\
Gender & 13 males, 12 females \\
\hline
\end{tabular}

tis, (2) a previous history of hip joint or knee surgery, and (3) hip joint instability. The experimental protocols and methods were explained in detail to all subjects, and all participants provided written informed consent in keeping with the ethical principles of the Declaration of Helsinki.

\section{Experimental methods}

The maximal isometric strength of the Gmed was quantified in side-lying position to assess the hip abductor strength using the dominant leg, as the leg preferred for kicking a ball. The strength measurement for the dominant side was performed as a tested hip side. To measure the strength of the Gmed muscle, the break test and the make test were performed in side-lying position. For the break test, a hand-held dynamometer (HHD, Lafayette Instrument Company, USA) was used. For the make test, maximal voluntary contraction strength of the Gmed muscle was measured with a tensiometer using a non-elastic band (Smart KEMA pressure sensor, Factorial Holdings Co., Korea).

The side for the measurement was defined on the opposite side from PD while subject was in one leg standing indicating the weak side of Gmed. ${ }^{4}$ The specific resistance region for the Gmed muscle was placed at the lateral malleolus in side-lying position. And a straight line at the same region on the skin was marked to minimize the regional difference. ${ }^{2}$ All measurements were performed on the same day to assess the test-retest reliability. The order of the tests between make and break test was randomized. Prior to the experimental procedure, the examiners and subjects were familiarized with the break and make tests to minimize measurement errors. The examiner provide support by holding ipsilateral pelvic iliac crest to minimize pelvic compensations (Figure 1). ${ }^{10}$ A tensiometer using a non-elastic band was used to measure the Gmed strength for make test. The examiner used the HHD to measure the Gmed strength with support by holding ipsilateral pelvic ilia crest for break test to minimize pelvic compensations (Figure 2). ${ }^{10}$ For the measurements, the knee joint in tested side was fully extended during 


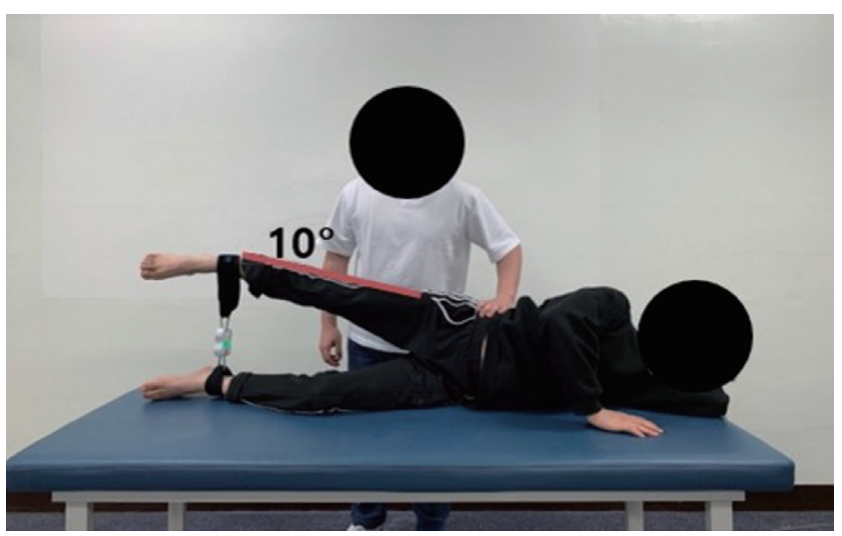

Figure 1. The make test to measure the strength of the gluteus medius in side-lying.

the isometric maximal voluntary contraction with 10 degrees of hip abduction. ${ }^{2,10}$ In addition, the hip and knee joint in non-tested side were performed in slight flexion to maintain the side-lying position. The duration of these contractions was approximately 5 seconds to measure the Gmed strength. The maximal strength provided by the tensiometer (in $\mathrm{kg}$ ) was retained. Each task was performed with 3 times and highest force was selected.

\section{Statistical Analysis}

All statistical analyses were conducted using SPSS software (ver. 21.0, SPSS, Inc., Chicago, IL, USA). Intra-class correlation coefficients (ICCs) with 95\% confidence intervals (CIs) were calculated. ${ }^{11}$ The ICC $(3,3)$ model was used to estimate intra-rater reliability of peak force (highest peak of 3 trials). In the interpretation, the ICC value of $>0.75$ was used to indicate "excellent," $0.40-0.75$ was "fair to good," and 0.00-0.40 was "poor". 12,13

\section{RESULTS}

The test-retest ICC of the strength measurement of Gmed for the make test $(0.90,0.79-0.98$ with $95 \%$ confidence interval, $18.35 \pm 2.68$ $\mathrm{kg}$ with averaged strength) was higher than that for the break test $(0.73,0.63-0.77$ with $95 \%$ confidence interval, $15.23 \pm 6.55 \mathrm{~kg}$ with averaged strength).

\section{DISCUSSION}

The purpose of the current study was to determine the test-retest reliability of make and break test of the strength measurement of

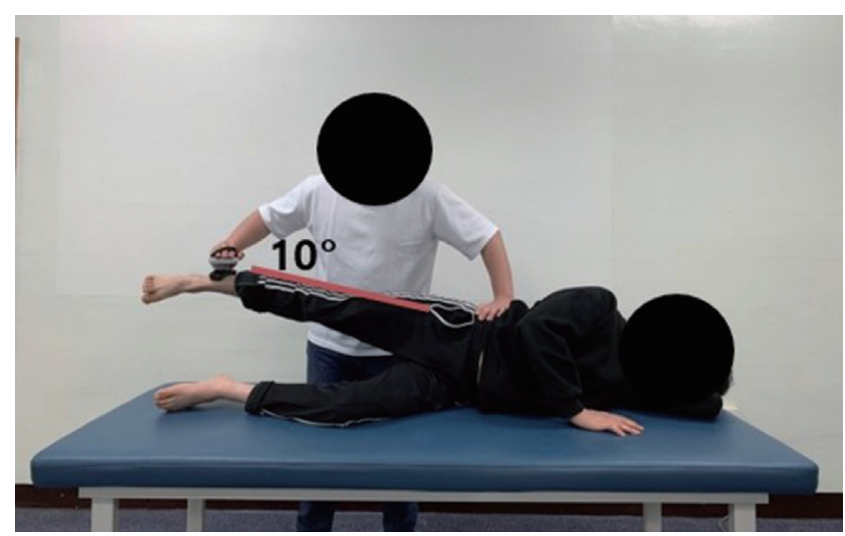

Figure 2. The break test to measure the strength of the gluteus medius in side-lying.

the Gmed in side-lying position in subjects with PD. We believe that the present research is the first reported study to investigate the testretest reliability of make and break test of the strength measurement of the weak Gmed in functional position like a side-lying. The results of this study showed that the test-retest ICC for the make test was higher than that of the break test in subjects with PD. The break test, which used an HHD, is the conventional way in clinical setting to measure muscle strength, whereas the make test, which used a tensiometer, is less common. These tests assess muscle contraction based on differences in resistance between isometric (make test) and eccentric contractions (break test).

There were some explanations to explain these findings. The fact that the make test measures strength by assessing isometric contractions may have contributed to its higher reliability compared to the break test in this study. A tensiometer using a non-elastic band was employed to maintain the hip abduction at a consistent angle of abduction in side-lying position. Although fatigue of the Gmed muscle occurred, the non-elastic band used for the make test may have contributed to maintaining the consistent abduction angle ${ }^{4}$ because the end position of hip abduction in side-lying position was controlled within the acceptable range of the band. In contrast, the break test using a HHD may have allowed variations in the abduction angle of hip joint in side-lying position depending on the Gmed performance, especially in subjects with PD. In addition, the strengths of the Gmed with break test was smaller than those of make test because of the length-tension relationship of insufficient performance of the Gmed muscle. ${ }^{4,7,8}$ The previous study reported that the electromyography (EMG) of Gmed was significantly increased and quadratus lumborum was significantly decreased with lumbar stabilization. ${ }^{3}$ Although 
the direct comparison of findings between the previous and this study was not possible, these results may indicate that the performance of Gmed can be related to core stability and neutral pelvic posture in side-lying especially in subjects with $\mathrm{PD} .^{3}$

In addition, the potential reason why the make test was more reliable than the break test to measure the Gmed strength with side-lying position was that possible adaptation in the constant task position. ${ }^{5,14}$ The measurement adaptation during make test was required for the subject's constant hip abduction angle in side-lying. However, the measurement adaptation during break test was required for both the examiner's experienced measurement skills and subject's consistent performance in same position. Even though all of the subjects were supported by the examiner's hand with pelvic iliac crest in side-lying during the test, a different test-retest reliability between the make and break test was shown. ${ }^{15}$ So, the results of this present study reported that the various elements that might affect the test-retest reliability should be controlled to minimize possible measurement errors. ${ }^{16}$

The equipment used for the make test has additional advantages, such as easy application and cost-effectiveness. These features make it more accessible in the clinical setting, in addition to minimizing possible measurement errors when evaluating Gmed strength due to consistency in the abduction angle of the hip joint in side-lying position. Therefore, the make test in side-lying position can be recommended to reliably measure Gmed strength in subjects with PD.

The current study had several limitations. First, the results cannot be generalized to elderly individuals. Further study is needed to investigate individual with elderly and neurologic impairments. Second, variations in the severity of PD were not considered in the strength measurements. Further study is needed to investigate the reliability difference of the strength measurement according to the severity of Gmed weakness. Third, the EMG was not employed to investigate the Gmed muscle. Further study is needed to investigate the EMG of the Gmed muscle during hip abduction in side-lying position. Fourth, the make test technique for hip abduction in subjects with PD cannot be generalized to the other muscles. Further study is needed to investigate test-retest reliability of make test for other muscle in various positions.

\section{ACKNOWLEDGEMENT}

This work was supported by the National Research Foundation of
Korea (NRF) grant funded by the Korea government (MSIT) (No. 2017R1C1B5076172).

\section{REFERENCES}

1. Sahrmann SA. Diagnosis and treatment of movement impairment syndromes. St Louis, Mosby, 2002.

2. Widler KS, Glatthorn JF, Bizzini M et al. Assessment of hip abductor muscle strength. A validity and reliability study. J Bone Joint Surg Am. 2009;91(11):2666-72.

3. Cynn HS, Oh JS, Kwon OY et al. Effects of lumbar stabilization using a pressure biofeedback unit on muscle activity and lateral pelvic tilt during hip abduction in sidelying, Arch Phys Med Rehabil. 2006;87(11):1454-8.

4. Trendelenburg F. Ueber den Gang bei angeborener Hüftgelenksluxation. Dstch Med Wochenschr. 1895:21(2):21-4.

5. Krause DA, Schlagel SJ, Stember BM et al. Influence of lever arm and stabilization on measures of hip abduction and adduction torque obtained by hand-held dynamometry. Arch Phys Med Rehabil. 2007;88(1):37-42.

6. Park JJ, So HJ, Shin WS. Effects of gluteus medius strengthening training using pressure biofeedback unit for muscle function and balance in stroke patients. J Kor Phys Ther. 2015; 27(4):221-7.

7. Schwartz S, Cohen ME, Herbison GJ et al. Relationship between two measures of upper extremity strength: manual muscle test compared to hand-held myometry. Arch Phys Med Rehabil. 1992;73:1063-8.

8. Agre JC, Magness JL, Hull SZ et al. Strength testing with a portable dynamometer: reliability for upper and lower extremities. Arch Phys Med Rehabil. 1987;68(7):454-8.

9. Arnold CM, Warkentin KD, Chilibeck PD et al. The reliability and validity of handheld dynamometry for the measurement of lower-extremity muscle strength in older adults. J Strength Cond Res. 2010;24(3):815-24.

10. Kendall KD, Schmidt C, Ferber R. The relationship between hip-abductor strength and the magnitude of pelvic drop in patients with low back pain. J Sport Rehabil. 2010;19(4):422-35.

11. Yoon TL. Validity and reliability of an inertial measurement unit-based 3D angular measurement of shoulder joint motion. J Kor Phys Ther. 2017;29(3):145-51.

12. Crossley KM, Bennell KL, Cowan SM et al. Analysis of outcome measures for persons with patellofemoral pain: Which are reliable and valid? Arch Phys Med Rehabil. 2004;85(5):815-22.

13. Koh EK, Jung DY. The reliability of flexor hallucis longus stretch test in subjects with asymmetric hallux valgus angles. J Kor Phys Ther. 2016;28(2):124-7.

14. Stratford PW, Balsor BE. A comparison of make and break tests using a hand-held dynamometer and the Kin-Com. J Orthop Sports Phys Ther. 1994;19(1):28-32.

15. McBeth JM, Earl-Boehm JE, Cobb SC et al. Hip muscle activity during 3 side-lying hip-strengthening exercises in distance runners. J Athl Train. 2012;47(1):15-23.

16. Desmyttere G, Gaudet S, Begon M. Test-retest reliability of a hip strength assessment system in varsity soccer players. Phys ThER Sport. 2019; 37(2):138-43. 